\title{
Airway epithelial CFTR mRNA expression in cystic fibrosis patients after repetitive administration of a recombinant adenovirus
}

\author{
Ben-Gary Harvey, ${ }^{1}$ Philip L. Leopold, ${ }^{1}$ Neil R. Hackett, ${ }^{1,2}$ Tina M. Grasso, ${ }^{3}$ \\ P. Mickey Williams, ${ }^{4}$ Ayly L. Tucker, ${ }^{4}$ Robert J. Kaner, ${ }^{1}$ Barbara Ferris, ${ }^{1}$ \\ Igor Gonda, ${ }^{4}$ Theresa D. Sweeney, ${ }^{4}$ Ramachandran Ramalingam, ${ }^{1}$ Imre Kovesdi, ${ }^{3}$ \\ Steven Shak, ${ }^{4}$ and Ronald G. Crystal ${ }^{1,2}$ \\ ${ }^{1}$ Division of Pulmonary and Critical Care Medicine, and \\ ${ }^{2}$ Belfer Gene Therapy Core Facility, Weill Medical College of Cornell University-New York Presbyterian Hospital, \\ New York, New York 10021, USA \\ ${ }^{3}$ GenVec Inc., Rockville, Maryland 20852, USA \\ ${ }^{4}$ Genentech Inc., South San Francisco, California 94080, USA \\ Igor Gonda’s present address is: Aradigm Inc., Hayward, California 94545, USA.
}

Address correspondence to: Ronald G. Crystal, Weill Medical College of Cornell University-New York Presbyterian Hospital, 520 East 70th Street, ST 505, New York, New York 10021, USA.

Phone: (212) 746-2258; Fax: (212) 746-8383; E-mail: geneticmedicine@mail.med.cornell.edu.

Received for publication July 22, 1999, and accepted in revised form September 22, 1999.

\begin{abstract}
We sought to evaluate the ability of an $\mathrm{E}^{-}, \mathrm{E}^{-}$adenovirus (Ad) vector ( $\left.\mathrm{Ad}_{\mathrm{GV}} \mathrm{CFTR} .10\right)$ to transfer the normal human cystic fibrosis transmembrane conductance regulator (CFTR) cDNA to the airway epithelium of individuals with cystic fibrosis (CF). We administered $\mathrm{Ad}_{\mathrm{GV}} \mathrm{CFTR} .10$ at doses of $3 \times 10^{6}$ to $2 \times 10^{9}$ plaque-forming units over 9 months by endobronchial spray to 7 pairs of individuals with CF. Each 3-month cycle, we measured vector-derived versus endogenous CFTR mRNA in airway epithelial cells prior to therapy, as well as 3 and 30 days after therapy. The data demonstrate that (a) this strategy appears to be safe; (b) after the first administration, vector-derived CFTR cDNA expression in the CF airway epithelium is dose-dependent, with greater than 5\% endogenous CFTR mRNA levels at the higher vector doses; (c) expression is transient, lasting less than 30 days; (d) expression can be achieved with a second administration, but only at intermediate doses, and no expression is observed with the third administration; and (e) the progressive lack of expression with repetitive administration does not closely correlate with induction of systemic anti-Ad neutralizing antibodies. The major advantage of an Ad vector is that it can deliver sufficient levels of CFTR cDNA to the airway epithelium so that CFTR expression protects the lungs from the respiratory manifestations of CF. However, this impressive level of expression is linked to the challenging fact that expression is limited in time. Although this can be initially overcome by repetitive administration, unknown mechanisms eventually limit this strategy, and further repetitive administration does not lead to repetitive expression.
\end{abstract}

J. Clin. Invest. 104:1245-1255 (1999).

\section{Introduction}

Cystic fibrosis (CF) is a common, recessive hereditary disorder caused by mutations in the cystic fibrosis transmembrane conductance regulator (CFTR) gene (1-3). The major manifestations are on the epithelial surface of the airways, with thick and sticky mucus, recurrent infections, and neutrophil-dominated chronic inflammation (4). The disease is associated with a progressive decline in lung function, with more than $90 \%$ of deaths secondary to pulmonary complications, at an average age of 31 years (5). There is extensive evidence that the pulmonary abnormalities in CF are initiated by a deficiency of CFTR function in the airway epithelium (1-6). With the demonstration that the normal CFTR cDNA could be transferred and expressed in the airway epithelium of experimental animals in vivo
(7), it was logical to hypothesize that this could be accomplished in the respiratory epithelium of individuals with CF (8). A number of clinical trials have demonstrated that this is feasible using adenovirus (Ad) (8-16), liposome/plasmid complexes (17-20), and adenoassociated virus vectors (21).

Now that the feasibility of human transfer of the normal CFTR cDNA to individuals with CF has been demonstrated, the next logical step in developing gene therapy for CF is to quantify the levels of gene transfer that can be achieved and for how long they persist. Analysis of CFTR mutation genotype/phenotype correlations in humans and mice suggests that persistent levels of $5-10 \%$ of normal CFTR expression evenly distributed throughout the airways should be sufficient to compensate for the deficiency of CFTR function result- 
ing from the parental CFTR mutations (22-27). Based on these considerations, the present study is directed toward determining whether it is possible to safely transfer and express the normal human CFTR cDNA delivered by an $\mathrm{E}^{-}, \mathrm{E}^{-} \mathrm{Ad}$ vector to the airway epithelium at levels greater than $5 \%$ of the endogenous CFTR mRNA levels. Using a study design in which an Ad vector expressing the normal CFTR cDNA $\left(\mathrm{Ad}_{\mathrm{GV}}\right.$ CFTR.10) is repetitively administered to individuals with $\mathrm{CF}$ by endobronchial spray every 3 months (for 3 cycles), 6 questions are addressed: (a) Is the vector dispersed evenly throughout the epithelium? (b) Are the levels of vector-derived CFTR mRNA achieved in the airway epithelium dosedependent, and what dose of the $\mathrm{Ad}_{\mathrm{GV}}$ CFTR.10 vector is necessary to achieve greater than $5 \%$ levels of normal CFTR mRNA in the airway epithelium after a single administration? (c) How long does the vector-derived CFTR cDNA expression persist? (d) Is repetitive administration safe? (e) Can vector-derived CFTR cDNA expression be achieved with repetitive administration, and how long does it persist? (f) Is there a correlation of the levels of airway epithelial expression of the vectorderived CFTR mRNA to the level of systemic anti-Ad neutralizing antibodies at the time of administration?

\section{Methods}

Study population. Fourteen individuals (12 male, 2 female, age $30 \pm 9$ years [mean \pm SEM; range, $17-48$ years]) were enrolled in the study. All had CF by conventional clinical criteria, including a positive sweat chloride test (4). Of the 14 individuals, 2 were $\Delta \mathrm{F} 508$ homozygotes, 10 were compound heterozygotes with one $\Delta \mathrm{F} 508$ allele, and 2 had mutations other than $\Delta \mathrm{F} 508$ in both alleles. All had mild to moderate lung disease typical of $\mathrm{CF}$, with an average forced expiratory volume in 1 second of $57 \pm$ $16 \%$ predicted (mean \pm SEM; range, $33-79 \%$ predicted).

Adenovirus vector. The $\mathrm{Ad}_{\mathrm{GV}}$ CFTR.10 vector, based on the subgroup $C$, serotype 5 genome, is missing E1a, most of E1b, and the majority of E3 sequences (Figure 1). $\operatorname{Ad}_{\mathrm{GV}}$ CFTR.10 contains an expression cassette that includes ( $5^{\prime}$ to $\left.3^{\prime}\right)$ : the cytomegalovirus early/immediate promoter/enhancer, an artificial splice sequence, the normal human CFTR cDNA, and SV40 stop/polyA sequences. The vector was produced, purified, and stored as described previously $(7,8)$. The final preparation $(1.6$ $\times 10^{11}$ plaque-forming units $[\mathrm{pfu}] \mathrm{mL}^{-1}$, particle $(\mathrm{pu})$ to pfu ratio 9 , and $3.7 \times 10^{10} \mathrm{pfu}^{-1}$, pu to pfu of 6) was demonstrated to have less than 1 replication-competent adenovirus in $2 \times 10^{9} \mathrm{pfu}$ (the maximal dose used) (8).

Table 1

Safety of endobronchial spray administration of the Ad $\mathrm{GV}_{\mathrm{C}} \mathrm{CF}$. 10 vector

\begin{tabular}{|c|c|c|c|c|c|c|c|c|c|c|c|c|c|}
\hline Study participant & 1 & 2 & 3 & 4 & 5 & 6 & 7 & 8 & 9 & 10 & 11 & 12 & 13 \\
\hline Dose $(p f u)^{A}$ & $10^{6}$ & $10^{6}$ & $10^{6.5}$ & $10^{6.5}$ & $10^{7}$ & $10^{7}$ & $10^{7.5}$ & $10^{7.5}$ & $10^{8}$ & $10^{8}$ & $10^{8.5}$ & $10^{8.5}$ & $10^{9}$ \\
\hline SymbolB & $\bigcirc$ & ○ & $\square$ & $\mathbf{\square}$ & $\diamond$ & $\bullet$ & $\square$ & - & $\square$ & D & 主 & & $\square$ \\
\hline \multicolumn{14}{|l|}{ Number of } \\
\hline administrations $^{C}$ & 3 & 3 & 1 & 3 & 1 & 3 & 3 & 1 & 2 & 3 & 3 & 3 & 3 \\
\hline \multicolumn{14}{|l|}{ Safety parameters ${ }^{D}$} \\
\hline Systemic & 0 & 0 & 0 & 0 & $2^{\mathrm{E}}, 1^{\mathrm{F}}$ & 0 & 0 & 0 & 0 & 0 & $1^{\mathrm{K}}$ & 0 & 0 \\
\hline Hematologic & 0 & 0 & 0 & 0 & 0 & 0 & 0 & 0 & 0 & 0 & 0 & 0 & 0 \\
\hline Coagulation & 0 & 0 & 0 & 0 & 0 & 0 & 0 & 0 & 0 & 0 & 0 & 0 & 0 \\
\hline Hepatic & 0 & 0 & 0 & 0 & 0 & 0 & 0 & 0 & 0 & 0 & 0 & 0 & 0 \\
\hline Gastrointestinal & 0 & 0 & 0 & 0 & $1^{\mathrm{H}}$ & 0 & 0 & 0 & 0 & 0 & 0 & 0 & 0 \\
\hline Neurologic & 0 & 0 & 0 & 0 & 0 & 0 & 0 & 0 & 0 & 0 & 0 & 0 & 0 \\
\hline Cardiovascular & 0 & 0 & 0 & 0 & 0 & 0 & 0 & 0 & 0 & $1^{\mathrm{J}}$ & 0 & 0 & 0 \\
\hline Pulmonary & 0 & 0 & 0 & 0 & 0 & 0 & 0 & $1^{\prime}$ & 0 & 0 & 0 & 0 & 0 \\
\hline
\end{tabular}

AFor each cycle, the actual dose was 3 times that listed, except for the $10^{9} \mathrm{pfu}$ dose, where the actual dose was 2 times that listed (see Meth-

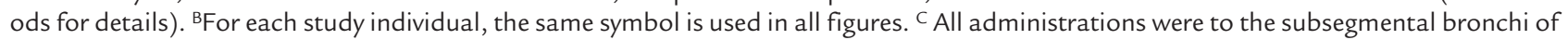
the right lower lobe; all individuals received 3 administrations of the vector, except for individuals 3, 5, 8, and 9 (see results for details). DNo adverse events wre attributable to the vector per se; the numbers are based on a graded toxicity scale: $0=$ none, $1=$ mild, $2=$ moderate, $3=$ severe, 4 = intolerable. ${ }^{E}$ Cycle 1, developed leukocytoclastic vasculitis with rash and microscopic hematuria 11 days following administration of vector. Individual had been receiving multiple antibiotics and had been bitten by a spider 2 days prior to vector administration. Syndrome was completely resolved with prednisone therapy within 3 months. Noanti-Ad antibodies were detected at any time. FTransient leukocytosis and anemia associated with fever related to bronchoscopic procedure. ${ }^{\mathrm{M}}$ Microscopic hematuria associated with vasculitis. ${ }^{\mathrm{H} B} \mathrm{Bloody}$ stool associated with prednisone treatment for vasculitis. 'Hypoxemia associated with narcotics used during bronchoscopy, resolved within 6 hours. JOccasional premature ventricular contractions noted on EKG monitor during bronchoscopy at 3 days post vector administration in cycle 2; no hemodynamic compromise; ectopies related to procedure discontinued after bronchoscopy was determined. ${ }^{\mathrm{K}}$ Transient leukocytosis and associated fever related to bronchscopy. 

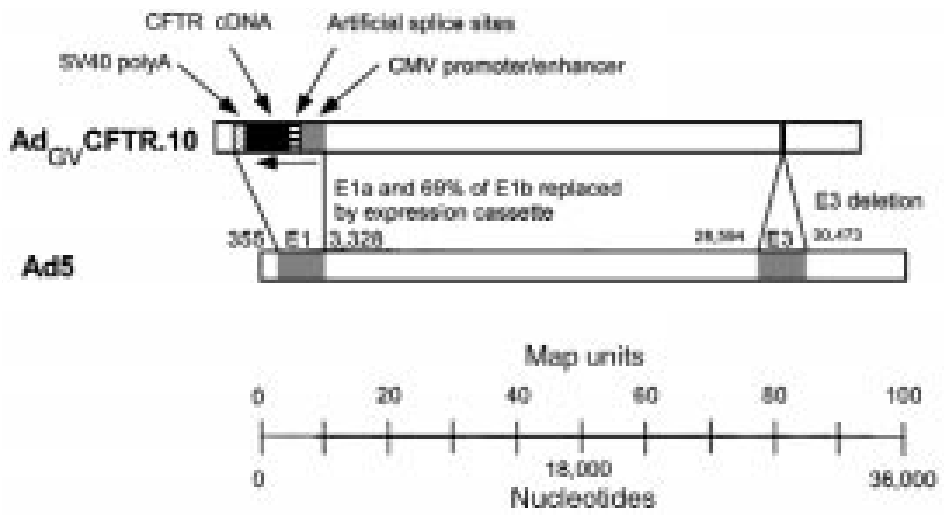

Figure 1

Schematic of the Ad ${ }_{\mathrm{GV}} \mathrm{CFTR} .10$ recombinant adenovirus vector. The E1a region and the majority of the E1b and E3 regions have been deleted. The expression cassette includes (right to left): the CMV enhancer/promoter, splice sequences, normal human CFTR CDNA, and the SV40 early polyadenylation signal. The deleted regions of E1 and $\mathrm{E} 3$ are indicated in map units.
Study design. The clinical study, approved by the local Institutional Review Boards and Biosafety Committees, the National Institutes of Health Recombinant DNA Advisory Committee, and The Food and Drug Administration, included 7 cohorts of 2 individuals each. The study started with a baseline period to ensure that the inclusion criteria were fulfilled, to establish baseline parameters for safety evaluation, to collect baseline airway epithelial cells, and to quantify serum anti-Ad5 neutralizing antibody titers (see below for details). This was followed by vector administration periods that were grouped into 3 cycles, each of 90 days, beginning at days 1, 91, and 181 , respectively. Each of the 7 dose cohorts were assigned a dose of the $\mathrm{Ad}_{\mathrm{GV}} \mathrm{CFTR} .10$ vector $10^{6}-10^{9} \mathrm{pfu}$ (in half-log increments). The vector was administered at days 1, 91, and 181 as a spray through a fiberoptic bronchoscope (see later here) to 2 sites within the lobar bronchus of the right lower lobe; e.g., the 2 individuals assigned the $3 \times 10^{6} \mathrm{pfu}$ dose received $10^{6} \mathrm{pfu}$ in 3 administrations to 2 sites within the lobar bronchus for a total dose of $3 \times 10^{6}$ pfu at each cycle beginning at 1,91 , and 181 days. For each cycle, airway epithelial cells (CFTR mRNA levels) and serum (for neutralizing antibodies) were obtained before vector administration and at days 3 and 30 after each vector administration (10). Assessment of the airway epithelial cells for dispersion of the $\mathrm{Ad}_{\mathrm{GV}}$ CFTR.10 vector was carried out only after the first cycle of the $3 \times 10^{8.5}$ pfu dose. Safety parameters, including hematological, serological, and urine studies, chest roentgenogram, pulmonary function tests, electrocardiogram (EKG), and assessment of shedding of vector (nasal, pharyngeal, rectal, blood and urine samples), were obtained at multiple times after vector administration at all dose levels (see later here).

Vector administration. An endobronchial spray delivery system was designed to ensure that the vector was deposited only on the airway epithelial surface and to an identifiable region that could be sampled over time (Figure 2). The vector was diluted in the storage buffer $(3 \%$ glycerol, $10 \mathrm{mM}$ Tris- $\mathrm{HCl}, 10 \mathrm{mM} \mathrm{MgC1} 2,150 \mathrm{mM} \mathrm{NaCl}$ [pH 7.8]) to the appropriate dose such that the dose to be delivered was in $100 \mu \mathrm{L}$. The fluid containing the vector was drawn into a catheter with a spray nozzle at one end (PW-6P washing pipe; Olympus America Inc., Melville, New York, USA). The actual volume drawn into the catheter was $120 \mu \mathrm{L} ; 100 \mu \mathrm{L}$ was delivered, and $20 \mu \mathrm{L}$ remained in the dead space. The catheter containing the vector was advanced through the working channel of a FB-15× fiberoptic bronchoscope (Pentax, Orangeburg, New York, USA) positioned just distal to the lobar bronchi of the right lower lobe. The formulation containing the vector was rapidly pushed through the spray nozzle with a 2-second, 55-psi pulse of air (1-2 mL) delivered through the proximal end of the catheter by an electronic actuating system (KDS824 Shot Meter; Kahnetics, Bloomington, California, USA) driving a syringe barrel; as the liquid is rapidly moved through the spray nozzle, the bulk liquid is broken into droplets. Ex vivo studies in casts of human bronchi, in vivo studies in rabbits, and in vivo studies in humans (normals and individuals with $\mathrm{CF}$ ) demonstrated that this system delivers the vector in a spray (droplet size distribution centered around $190 \pm 9 \mu \mathrm{m}$ volume median diameter; < $1 \%$ particles $<10 \mu \mathrm{m}$ diameter) to the targeted segmental bronchi in a circumferential fashion over an area from just distal to the nozzle for up to 2 bifurcations, i.e., 3-4 $\mathrm{cm}$ of the distal bronchi (28). Videotapes were made of

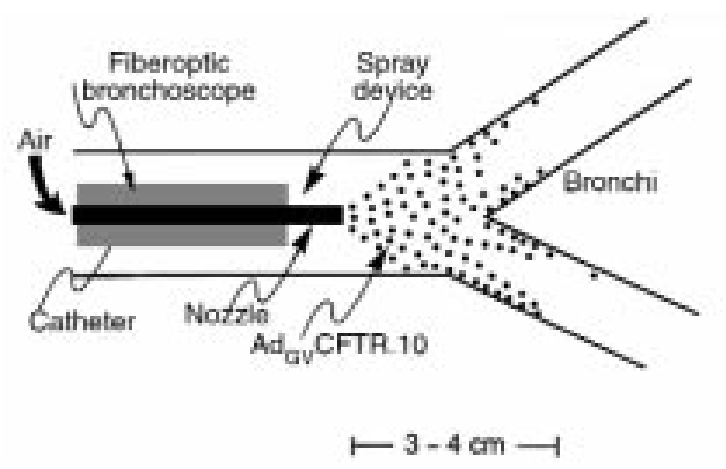

\section{Figure 2}

Strategy used to spray the $\mathrm{Ad}_{\mathrm{GV}} \mathrm{CFTR} .10$ vector onto the airway epithelium. The vector is diluted to the required dose, and the solution is drawn into the catheter. The catheter is inserted in the channel of a fiberoptic bronchoscope previously placed in the lobar bronchus. The catheter/nozzle delivers the vector in a volume of 100 $\mu \mathrm{L}$ as a spray (droplet size, $190 \mu \mathrm{m}$ ) to the target area of the bronchi with the aid of an electronic actuating system that drives a syringe barrel over 2 seconds, pushing a column of air $(1-2 \mathrm{~mL})$ that generates the spray uniformly coating the epithelium over $3-4 \mathrm{~cm}$. 


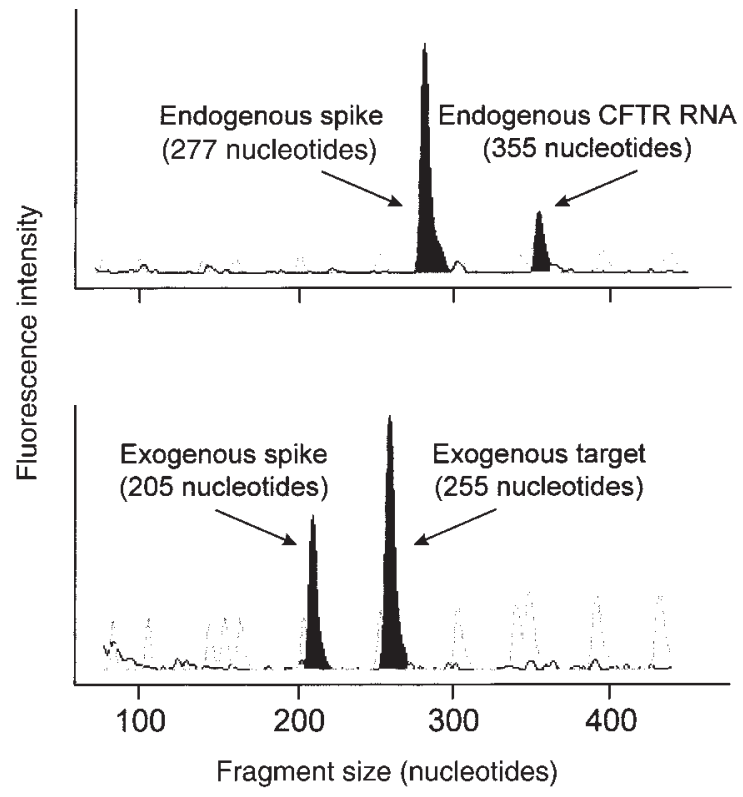

Figure 3

Competitive PCR analysis used to quantify the amount of exogenous (vector-derived) CFTR mRNA and endogenous CFTR mRNA in airway epithelial cells. Using nested RT-PCR using fluorescent primers, RNA samples prepared from bronchial brushings were spiked with standard RNAs at concentrations comparable to the endogenous or exogenous levels estimated from preliminary analyses. The spike for the endogenous RNA is derived from in vitro transcription of a plasmid containing the CFTR mRNA with a 78-bp deletion. The spike of the exogenous RNA is derived from in vitro transcription of a plasmid containing the cloned Ad-encoded CFTR mRNA with a 50-bp deletion. These competitor RNAs were shown to amplify with equal efficiency as the target of interest (data not shown). The RNA mixtures were subjected to nested PCR as described in Methods with 1 of the second-round primers labeled with FAM. The PCR products were identified from fluorescent electropherograms by their mobility relative to rhodamine-labeled molecular weight standard and quantified using the GeneScan software (Perkin-Elmer Applied Biosystems). The upper panel shows detection of the endogenous CFTR mRNA and spike, and the lower panel shows an example of detection of exogenous CFTR mRNA and spike.

each delivery site, along with appropriate notes as to anatomic location; these were reviewed before each bronchoscopy to ensure that correct locations were identified.

Quantitative assessment of CFTR $m R N A$. Airway epithelial cells were collected with multiple brushings through a fiberoptic bronchoscope as described previously $(8$, 29). Bronchoscopies for collection of airway epithelial cells were performed at baseline (before vector administration) and after each vector administration, at days 3 and 30. During each bronchoscopy, a brush was advanced through the working channel of the bronchoscope, and airway epithelial cells from areas that received the vector were obtained by gliding the brush several times over the airway surface. The cells obtained were immediately flicked in a $15-\mathrm{mL}$ conical tube containing LHC-8 medium (BioFluids, Rockville, Maryland, USA) and kept at $4^{\circ} \mathrm{C}$ until processing. Cell number was quantified in a hemocytometer, and differential cell counts were obtained using modified Giemsa stains (Dade International, Miami, Florida, USA) of cytospin preparations (29). Of 120 samples analyzed, $8.4 \times 10^{6} \pm$ $0.5 \times 10^{6}$ total cells (mean $\pm \mathrm{SEM}$; range, $1.4 \times 10^{6}$ to 2.5 $\times 10^{7}$ ) were obtained on the average, with $65 \pm 2 \%$ bronchial epithelial cells (range, 22-97, except for 5 samples that yielded $<20 \%$ epithelial cells); the remainder were inflammatory cells, of which $94 \pm 2 \%$ were neutrophils (range, 80-100, except for 4 samples that yielded $0 \%$ neutrophils and $100 \%$ macrophages).

Quantitative, competitive RT-PCR (QC-RT-PCR) was used to assess the amount of vector-derived CFTR mRNA (referred to as "exogenous CFTR mRNA") in the samples (30). All assessments were carried out in blinded fashion by individuals not involved with the clinical aspects of the study. The code was broken twice during the study: after the $10^{8}$ pfu dose was reached, and at the completion of the study. Because the samples were a variable mixture of bronchial epithelial cells and inflammatory cells, the amount of exogenous CFTR mRNA was referenced to the amount of endogenous (i.e., the subject's own CFTR mRNA) in the sample. Prior studies have demonstrated that the levels of CFTR mRNA in the airway epithelium recovered by brushing is 1-2 mRNA copies per cell, with normal and CF CFTR mRNA levels similar (31). In contrast, inflammatory cells have extraordinarily low levels of CFTR mRNA; i.e., in the airways, CFTR mRNA levels are a marker of epithelial cells (32).

To obtain "total" RNA from the brushed cell samples, a portion of the PBS washed cell pellet (approximately $3 \times 10^{6}$ cells) was lysed immediately and stored frozen at $-70^{\circ} \mathrm{C}$. The frozen cell lysates were thawed, and total RNA was purified (Micro-Scale Total RNA Separator; CLONTECH Laboratories Inc., Palo Alto, California, USA). The RNA was dissolved in TE (10 mM Tris, 1 mM EDTA [pH 8.0]).

The presence of vector-derived (exogenous) and endogenous CFTR mRNA was assayed by RT-PCR using nested primers. Primers were designed that selectively amplify RNA and exclude contaminating vector or genomic DNA. An initial screening evaluation was carried out to determine whether exogenous Ad-encoded mRNA was present. This screening was performed on 3 replicates of all samples. Three aliquots of RNA were reverse transcribed using AMV RT and then subjected to 40 cycles of PCR using Tfl polymerase (RTAccess Kit; Promega Corp., Madison, Wisconsin, USA). The primers used are shown here, with a forward slash in the sequence of the Ad-encoded CFTR 5' primer showing where the intron is excised from the mRNA: (a) Ad-encoded CFTR (forward, outer): CCG TGC CAA GAG TGA C/GT GTC; (b) Ad-encoded CFTR (reverse, outer): AAG CCA GCT CTC TAT CCC ATT CTC; (c) endogenous CFTR (forward, outer): CAG GCA CCC AGA GTA GTA GGT C; and (d) endogenous CFTR (reverse, outer): CTG TAC TGC TTT GGT GAC TTC C. An aliquot $(1 \mu \mathrm{L})$ of the product was used for a second round of PCR using the Taq DNA polymerase and a sec- 


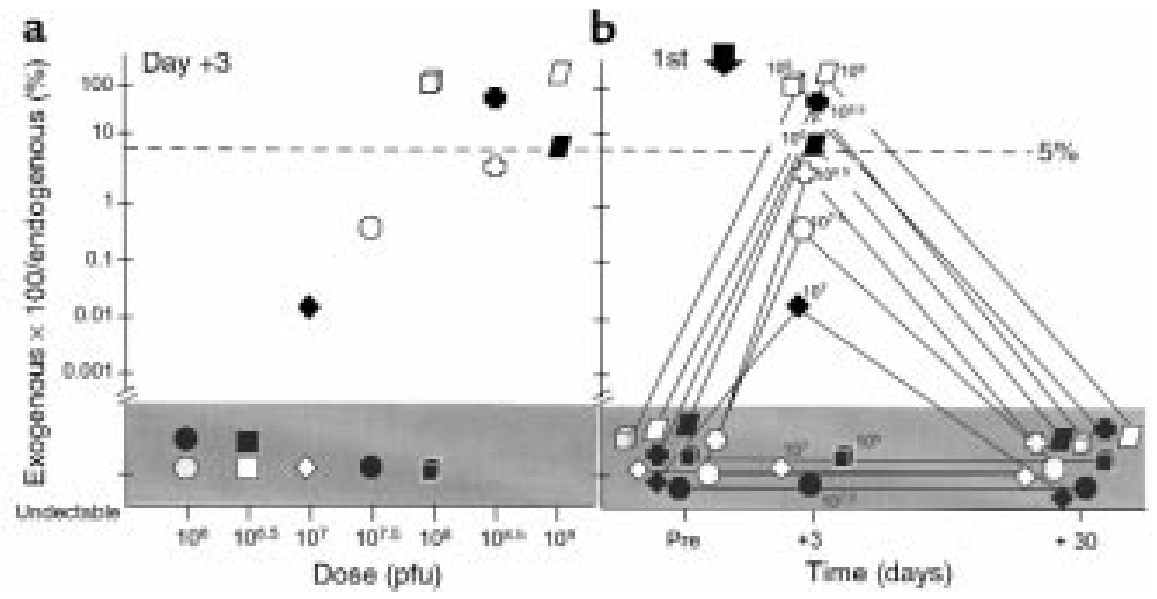

\section{Figure 4}

Quantitative assessment of the airway epithelium for the percentage of exogenous CFTR mRNA (derived from the Ad $\mathrm{GV}$ CFTR.10 vector) compared with endogenous CFTR mRNA (individual's own CFTR gene expression) as a function of dose and time (baseline, days 3 and 30) after endobronchial spray of the first administration (cycle 1) of the $\mathrm{Ad}_{\mathrm{GV}} \mathrm{CFTR} .10$ vector. The dashed line represents the $5 \%$ level of exogenous vector-derived CFTR mRNA. Each symbol represents a different individual (see Table 1). (a) Function of dose at day 3. (b) Function of time. The data for the $10^{6}$ and $10^{6.5}$ doses (undetectable at each time point for all subjects) are not shown in $\mathbf{b}$. "Pre" is before first vector administration.

ond primer set, 1 of which was labeled at the $5^{\prime}$ end by 6-( $N$-hexyl)-fluoresceinamide (FAM): (a) Ad-encoded CFTR (forward, nested): TGA C/GT GTC CAC TCC CAG G; (b) Ad-encoded CFTR (reverse, nested): FAMTCA GCA GAA TCA ACA GAA GGG; (c) endogenous CFTR (forward, nested): TTT GGC ATT AGG AGC TTG; and (d) endogenous CFTR (reverse, nested): FAMGCT GTA CTG CTT TGG TGA CT. Aliquots of the second $\mathrm{PCR}$ reaction were run on a $4.5 \%$ denaturing gel on a DNA sequencer (model 373A; Perkin-Elmer Applied Biosystems, Foster City, California, USA) under the GeneScan software (Perkin-Elmer Applied Biosystems). Each of the 3 samples was inspected for product of the expected size. If positive in at least 2 of the 3 replicates, the sample was considered valid (i.e., positive for exogenous Ad-CFTR mRNA expression). The fluorescent signal intensity generated by the PCR product of each positive result was compared with signals obtained from a serial dilution of known amounts of target to estimate the levels of exogenous and endogenous CFTR mRNA found in each sample. This estimate was used as a guide for how much spike (i.e., competitor) would be needed for the subsequent quantitative competitive RT-PCR analysis described later here. Spike RNAs were designed to be specific for both the Ad-vector-derived and endogenous CFTR mRNAs. The RNAs contained the specific sequences that were amplified in the nested RTPCR protocols, including both outer and nested primer binding sites. A small restriction fragment was excised from both the Ad-vector exogenous and endogenous CFTR PCR products. This was cloned into plasmids downstream of T7 RNA polymerase sites. T7 RNA polymerase was used to generate the spike RNA molecules. Upon RT-PCR amplification of these spikes, their smaller product size was easily discriminated from the actual target products (Figure 3). Each spike was tested and demonstrated to amplify with equal efficiency com- pared with its matched target. QC-RT-PCRs were then run on sample replicates of all positive samples. Replicate reactions were performed using at least 2 known concentrations (as already estimated here) of the spike RNA added to the sample (Figure 3). Endogenous and vector-derived quantitative competitive RT-PCR reactions were performed with spikes of known concentration, and the amount CFTR mRNA was calculated from the ratio of the fluorescent intensity of amplification products. The final data were expressed as: exogenous CFTR mRNA $\times 100 /$ endogenous CFTR mRNA. Each RT-PCR contained RNA derived from approximately 5,000 airway epithelial cells. The sensitivity of the assay was at least $10^{-5}$ copies of exogenous CFTR mRNA per cell, and the coefficient of variation of the assay was $20 \%$. By using internal RNA standards of concentration approximating those of the sample, the linear range was more than $10^{5}$-fold. All samples were evaluated in duplicate, and the 2 values were averaged.

Fluorescence in situ bybridization (FISH). To assess the airway epithelial cells for dispersion of the $\mathrm{Ad}_{\mathrm{GV}}$ CFTR.10 vector in the airway nuclei, bronchial brushings from individuals receiving $3 \times 10^{8.5} \mathrm{pfu}$ of the $\mathrm{Ad}_{\mathrm{GV}}$ CFTR.10 vector were analyzed by in situ hybridization according to the methods of Gussoni et al. (33). Probes for the Ad genome (200-600 bp) were prepared from an AdNull vector (34) by nick translation (Boehringer Mannheim Biochemicals Inc., Indianapolis, Indiana, USA). The digoxigenin-labeled probes were precipitated with fluorescein-labeled human chromosome 7 DNA (Boehringer Mannheim Biochemicals Inc.) before hybridization. Parallel samples of bronchial brushings were assessed by immunocytochemistry for expression of cytokeratin-18 (35) using FITC-conjugated anti-cytokeratin 18 (Sigma Chemical Co., St. Louis, Missouri, USA) or, as a control, FITC-conjugated CD4. 
Neutralizing antibodies. Anti-Ad5 neutralizing antibody titers were measured by the ability of serum to prevent infection of A549 cells (CCL185; American Type Cell Culture Collection, Rockville, Maryland, USA) by wildtype Ad5 as described previously $(10,36,37)$.

Safety. All individuals were tested once in the baseline period for CF genotype, pregnancy, and human immunodeficiency virus antibodies. Chest roentgenogram, pulmonary function tests, and EKG were done at baseline and once within 3 days after vector administration. Complete blood count, erythrocyte sedimentation rate, serum electrolytes, clotting factors, liver function tests, urine analysis, and serum anti-Ad5 antibody titer were done at baseline and at days 1, 2, 3, 30, and 60 days after vector administration. Cultures were done of nasal, pharyngeal, sputum, urine, and blood samples for replication-deficient and replication-competent Ad at baseline and for 3 days after vector administration. Adverse events were recorded and assessed regarding the link, if any, to the Ad vector.

Statistical evaluation. Data relating to baseline characteristics of the study population are presented as mean \pm SEM. Given that this is a phase I clinical trial, the number of patients at each dose $(n=2)$ and the total number of patients $(n=14)$ are too small to provide sufficient statistical power to discriminate within the variability of the various parameters that were assessed. Therefore, lack of statistical significance should not necessarily be interpreted as "no difference." In this context, the results are presented without formal error estimates and are presented in the context of trends suggested by the data.

\section{Results}

General. Of the 14 individuals enrolled, 10 completed the full study (e.g., all 3 cycles of vector administration). Of the 4 individuals not completing the study, 1 was discontinued after the second cycle because of the devel- opment of arrhythmias during a bronchoscopy procedure, and 1 was discontinued 11 days after the first vector administration because of the development of leukocytoclastic vasculitis (see later here). Two individuals dropped out for personal reasons. To follow each individual through the entire data set, the same symbol is used for the same individual throughout (see Table 1). Safety. The study was carried out without any significant adverse effects clearly attributable to the Ad vector (Table 1). One individual (dose, $3 \times 10^{7} \mathrm{pfu}$ ) developed leukocytoclastic vasculitis with bilateral lower extremity rash and microscopic hematuria, 11 days after vector administration. No pulmonary abnormalities were observed. This individual was receiving a variety of medications for $\mathrm{CF}$ and was bitten by a spider 2 days previously. Neither replication-competent nor replication-deficient virus was observed in nasal, pharyngeal, rectal, blood, or urine samples obtained at multiple times after vector administration. No rise in anti-Ad antibodies was detected over a 3-month observation period. Although it is not possible to definitively exclude the vector as an etiological agent, the lack of anti-Ad antibodies or of Ad as a persistent antigen suggests that this event was not associated with administration of the vector.

No shedding of the vector was detected in any subject at any time during the study. Assessment of nasal, pharyngeal, urine, blood, and rectal samples at days 1-3 after vector administration showed no detectable replication-deficient or replication-competent adenovirus in a total of 345 samples.

Vector dispersion in the airway epithelium. Cytospin preparations of bronchial brushings included intact islands of cells, single intact cells, broken cells, debris, and mucus as described previously (29). Intact islands of epithelial cells contained well-preserved nuclei and closely apposed cell membranes. Single cells included a mixture of epithelial cells and inflammatory cells, more
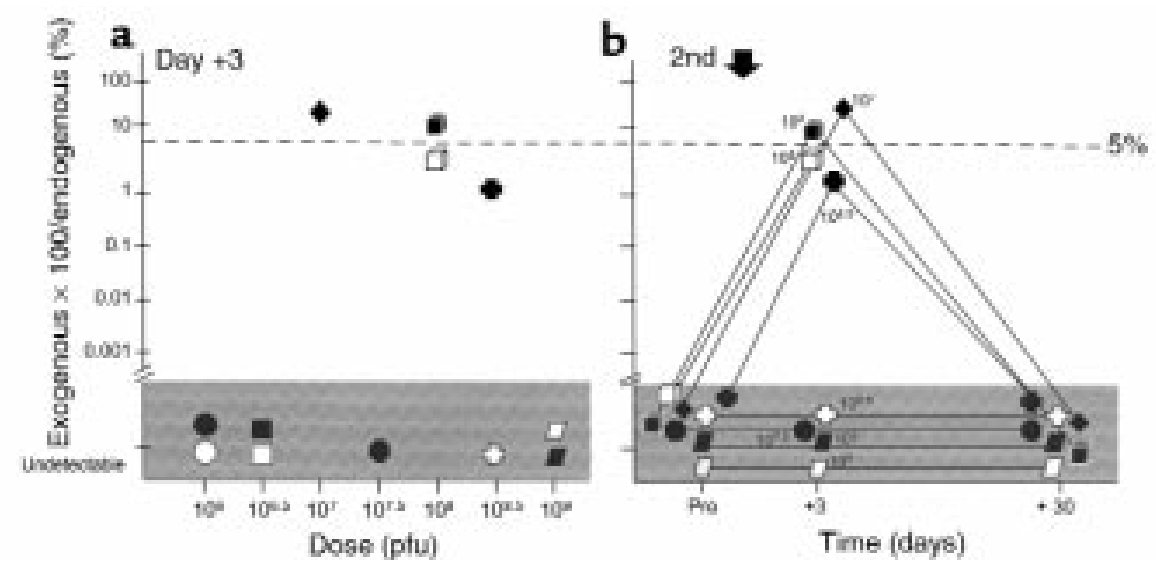

Figure 5

Quantitative assessment of the airway epithelium for the percentage of exogenous CFTR mRNA compared with endogenous CFTR mRNA as a function of dose and time (baseline, days 3 and 30) after endobronchial spray of the second dose (cycle 2) of the Ad GVCFTR.10 vector. The dashed line represents the $5 \%$ level of exogenous vector-derived CFTR mRNA. Each symbol represents a different individual (see Table 1). (a) Function of dose at day 3. (b) Function of time. "Pre" is day 90 of the study. The data for the $10^{6}$ and $10^{6.5}$ doses (undetectable at each time point for all subjects) are not shown in $\mathbf{b}$. 


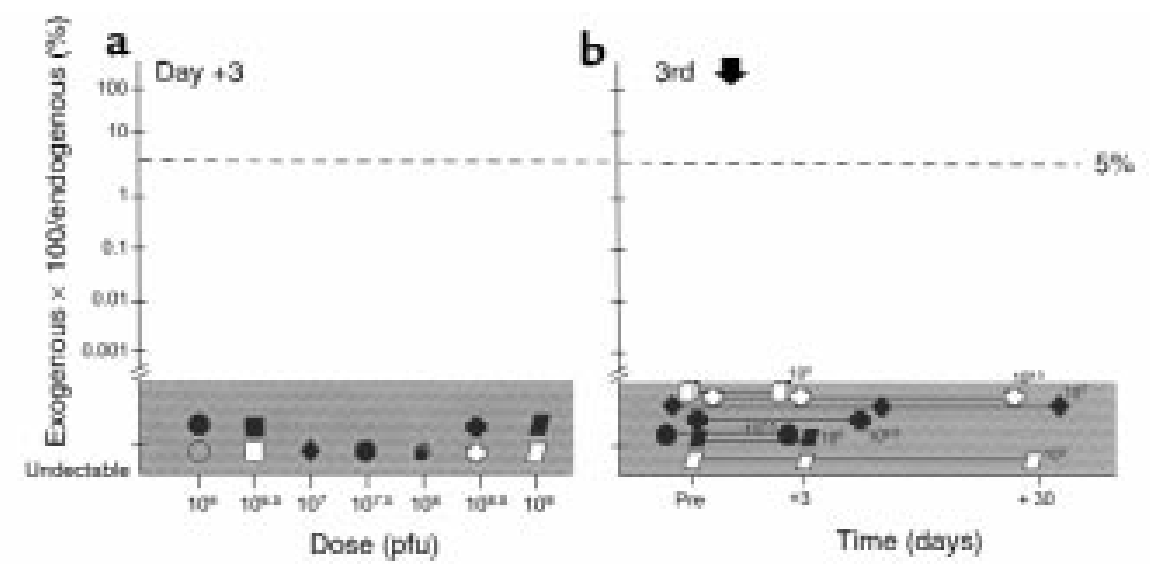

\section{Figure 6}

Quantitative assessment of the airway epithelium for the percentage of exogenous CFTR mRNA compared with endogenous CFTR mRNA as a function of dose and time (baseline, days 3 and 30) after endobronchial spray of the third dose (cycle 3) of the vector. The dashed line represents the $5 \%$ level of exogenous vector-derived CFTR mRNA. Each symbol represents a different individual (see Table 1). (a) Function of dose at day 3. (b) Function of time. "Pre" is day 180 of the study. The data for the $10^{6}$ and $10^{6.5}$ doses (undetectable at each time point for all subjects) are not shown in $\mathbf{b}$.

than $93 \%$ of which were neutrophils, with the remaining cells being alveolar macrophages. A significant proportion of single cells were not viable $(41 \pm 2 \%$; range, 8-70). Based on the concept that the epithelial cells in the islands provided a reasonable representation of the epithelium in situ and could provide an estimate of the dispersion of the vector in the intact epithelial sheet, all FISH analyses were carried out in the island population of epithelium. Intact islands of cells in cytospin preparations of bronchial brushings were positive for cytokeratin-18, showing that the islands corresponded to nondissociated patches of airway epithelium.

All probes used in the analysis of Ad genome in patient samples were tested for specificity by conducting control experiments in parallel with each analysis of patient samples. Controls, including human A549 lung epithelial carcinoma cells infected with AdNull in vitro, normal human airway epithelial cells from bronchial brushings infected with AdNull in vitro, and naive cell samples for each cell type, were positive for human chromosome 7 and, when infected with AdNull, were positive for Ad vector. In A549 cells, the assay was able to detect at least 2 copies of Ad genome per cell.

Despite these controls, we were unable to identify definitively the Ad vector genome in the clinical samples using FISH analysis. Although signal of the appropriate wavelength (red channel) was observed in a dispersed fashion in the epithelial cells throughout the sample, the signals were low level and could not be definitively identified as different from low-level autofluorescence that was observed specifically at red wavelengths in the brushed airway epithelial clinical samples from both CF and normal individuals. Although these data are strongly consistent with the concept that the vector is not localized at high levels in small foci of cells, the autofluorescence did not permit a definitive statement regarding demonstration that the vector was dispersed evenly throughout the epithelium.
First administration. To evaluate the level of vectorderived CFTR mRNA in the airway epithelium of naive individuals (e.g., first-dose Ad vector) after endobronchial spray administration of $\mathrm{Ad}_{\mathrm{GV}}$ CFTR.10, airway epithelial samples were evaluated before, and at days 3 and 30 after, vector administration. No vector-derived CFTR mRNA was detected in any pretherapy samples except for individual 8 (cycle $1,3 \times 10^{7.5}$ pfu dose). This was the only false positive in a total of 40 pretherapy samples for all cycles $(n=14$ for cycle 1 ; $n=11$ for cycle $2 ; n=10$ for cycle 3 ), and it was concluded that the "pre" and " +3 day" samples for this individual had been, most likely, inadvertently mixed. However, because this could not be definitely proved, all samples for cycle 1 of individual 8 were eliminated from the analysis.

Evaluation of the cycle $1,+3$ day samples showed a dose dependence in vector-derived CFTR mRNA expression in the airway epithelium (Figure 4a). No vector-derived expression was observed in any individuals at 3 days at the $3 \times 10^{6}$ to $3 \times 10^{6.5}$ pfu dose range. In contrast, positive vector expression was observed in all individuals at the $3 \times 10^{8.5}$ to $3 \times 10^{9} \mathrm{pfu}$ dose range. In the mid-dose range $\left(3 \times 10^{7}\right.$ to $\left.3 \times 10^{8} \mathrm{pfu}\right)$, some individuals demonstrated vector-derived CFTR mRNA expression and some did not. Strikingly, not only was dose-dependent expression observed, but at the higher dose ranges, most of the samples demonstrated levels of vector-derived CFTR mRNA at or above the $5 \%$ level of exogenous CFTR mRNA compared with the level of endogenous CFTR mRNA believed to be in the therapeutic range (22-27). Re-evaluation of the airway epithelium of the same individuals 30 days after the first vector administration demonstrated no exogenous gene expression in any of the study individuals (Figure 4b). Because airway epithelial samples were not gathered at time points between 3 and 30 days, it is not possible to come to a conclusion regarding the persist- 


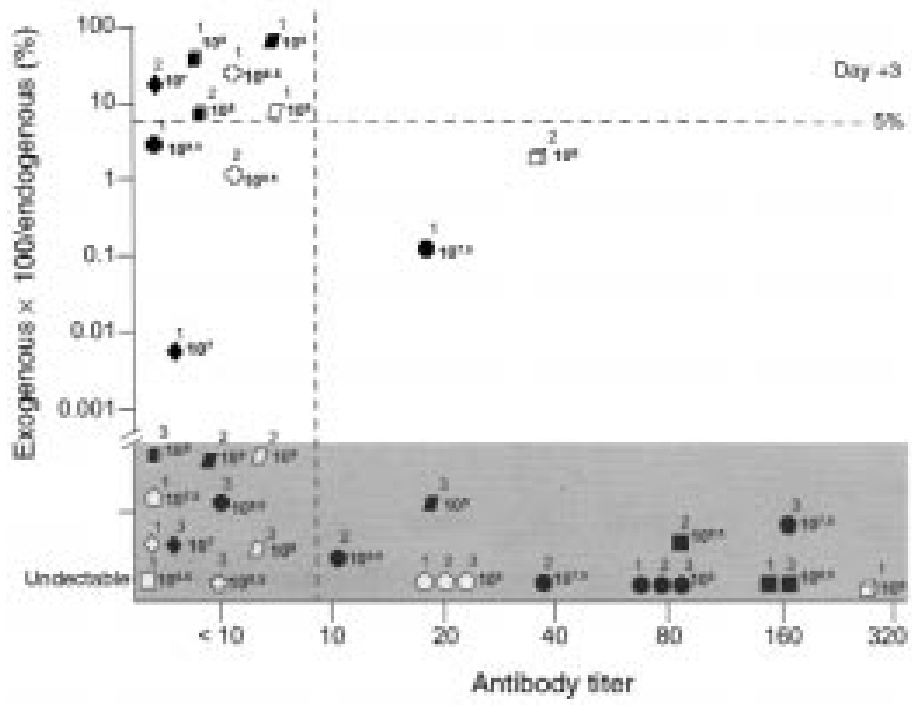

\section{Figure 7}

Comparison of the quantitative assessment of the exogenous and endogenous CFTR mRNA at day 3 (all cycles) to the serum anti-Ad5 neutralizing antibody titer at day 1 of each cycle (the time of administration of the vector). Each symbol represents a different individual (see Table 1). The dose (in pfu) is next to each symbol (or group of symbols, if relevant); the number above each symbol represents the cycle number. The horizontal dashed line represents the $5 \%$ level of exogenous vector-derived CFTR mRNA. The vertical dashed line represents the detectable limit of neutralizing anti-Ad antibodies. The pretherapy neutralizing antibody data has been published previously (10). ence of expression over this period. However, we can conclude from these studies that: (a) at high doses, vector-derived expression 3 days after administration is at or above normal levels; and (b) the expression does not persist for 30 days, and thus, in its current form, Ad vectors are incapable of maintaining normalizing levels of vector-derived CFTR mRNA levels in the airway epithelium on a persistent basis after a single administration.

Second administration. At the lower doses $\left(3 \times 10^{6}\right.$ to 3 $\left.\times 10^{6.5} \mathrm{pfu}\right)$ of the $\mathrm{Ad}_{\mathrm{GV}} \mathrm{CFTR} .10$ vector, repeat administration at the second cycle (initiated 90 days after the first administration) demonstrated no expression (Figure 5). Interestingly, for the intermediate doses, expression was observed, with levels at or above the $5 \%$ "protective" level (Figure 5a). However, in marked contrast to the first administration, the $3 \times 10^{9}$ pfu dose yielded no expression with the second administration. Thus, although expression is achieved at some doses with the second administration, it is not dose-dependent, as with the first administration. Rather, it appears that midlevel doses yield repeat expression, but repeated high-level doses do not yield measurable expression. As observed in the first cycle of vector administration, for those dose levels at which expression was observed 3 days after the second administration, expression was back to baseline by day 30 (Figure 5b). Thus, although samples were not available to determine whether expression persisted at 4-29 days, it clearly does not persist to 30 days.

Third administration. Unlike observations after the first and second administrations, administration of the vector the third time resulted in no expression of vector-derived CFTR mRNA in the airway epithelium (Figure 6). This was true at all dose levels (Figure 6a) and at both days 3 and 30 after the third vector administration (Figure 6b).

Systemic anti-Ad antibodies. One explanation for the inability to have successful gene transfer by the third administration (Figure 6), and the inability to have successful gene transfer at the highest doses after the second administration (Figure 5), is that the intrabronchial administration of the Ad vector induces systemic antiAd5 neutralizing antibodies that prevent the vector from successfully infecting the airway epithelial target cells. On the basis of studies in experimental animals (36-39), we expected that serum neutralizing antibodies would be observed, at least at higher doses and/or with repeat administration of the vector. As previously reported as part of an analysis of systemic anti-Ad neutralizing antibody titers in association with several human gene therapy applications, there were no major increases in serum anti-Ad5 neutralizing antibodies in any individual in the present study (10). An occasional sample was observed with a 2- to 4-fold increase over the baseline level, but this was not dose-dependent. In some instances, the antiAd5 neutralizing antibody levels actually decreased after vector administration. Although these observations do not yield insight regarding antibody levels on the airway epithelial surface, they clearly demonstrate that repetitive endobronchial administration of an Ad5 vector to individuals with CF does not induce significant levels of systemic anti-Ad5 neutralizing antibodies.

Interestingly, a comparison of vector-mediated expression at day 3 after vector administration to the level of serum anti-Ad5 neutralizing antibodies at the time of vector administration (Figure 7 ) showed that at anti-Ad titers of $\leq 1: 40$, there was no relationship between the level of expression from the vector and the serum anti-Ad neutralizing antibody titer. However, at anti-Ad titers $\geq 1: 80$, no vector-derived expression was observed, regardless of dose or whether it was the first, second, or third administration.

\section{Discussion}

Since the initial demonstration by Rosenfeld et al. (7) that it was possible to use a replication-deficient recombinant adenovirus vector to transfer the normal CFTR cDNA to the respiratory epithelium of experimental animals, there have been a number of studies demon- 
strating that it was feasible to do this in humans (8-16). The present study was designed to take the next step in the development of this therapy by asking whether it is feasible to use an Ad gene transfer vector to safely mediate levels of normal CFTR mRNA believed to be sufficient to correct this disorder, and, if so, how long did the expression last and whether persistence could be achieved with repetitive administration.

Studies in experimental animals have suggested that Ad vector-mediated gene transfer and expression in the airway epithelium is feasible and can achieve high levels of gene transfer, but that expression is transient and repeat administration is limited by neutralizing antibodies directed against the Ad capsid (36-39). Using an E1-, E3- Ad vector to transfer and express the normal human CFTR cDNA in the bronchial epithelium after repetitive endobronchial administration to individuals with $\mathrm{CF}$, and quantitative PCR analysis of vector-derived expression of normal CFTR mRNA in the airway epithelium, the present study demonstrates that normal CFTR mRNA levels can be safely achieved in the airway epithelium of individuals with CF, but that the general paradigm of limited persistence of vector expression, derived from studies in experimental animals, is also valid for humans with $\mathrm{CF}$. The mechanisms underlying the lack of persistence of the vector-derived gene expression in the airways are unknown. However, as expression diminishes with repetitive administration, it is likely that antiAd adaptive immune mechanisms are involved, despite the inability of repetitive administration to induce systemic anti-Ad neutralizing antibodies (10).

Safety. In our original report demonstrating the feasibility of Ad vector CFTR cDNA transfer to the human airway epithelium, we observed in 1 individual receiving $2 \times 10^{9}$ pfu of the vector, a transient syndrome of fever, infiltrates on the chest roentgenogram and computerized tomographic scan, and hypotension in association with increased levels of IL- 6 in the $\operatorname{serum}(8,40)$. In that trial, the vector was delivered in a volume of 20 $\mathrm{mL}$, a volume that we now recognize must have delivered some vector to the alveolar epithelium. In the present study, the vector was delivered in a total volume of $100 \mu \mathrm{L}$ using a bronchial spray device that limited more than $95 \%$ of the vector to a limited segment of the airways (28). With this delivery strategy, doses of $2 \times 10^{9}$ pfu were repetitively administered (every 3 months for 3 cycles) without adverse effects. Consistent with this observation, we have used the same strategy to administer a vector of the same design to the airway epithelium of normal individuals with similar doses with no adverse sequelae (41). Thus, when delivery of the Ad vector was limited to the airway epithelium, severe adverse events were not observed.

Levels of vector-derived normal CFTR $m R N A$ in airway epithelium. Compared with all other gene transfer vector systems available for human use, the major advantage of an Ad vector is that it can deliver and express the CFTR cDNA to the airway epithelium with levels at or above those believed to be protective of developing pulmonary abnormalities in individuals with CF (22-27). In this regard, after the first administration, vector-derived CFTR cDNA expression in the CF airway epithelium is dose-dependent, with levels of greater than $5 \%$ endogenous CFTR mRNA levels achieved at the higher vector doses. The 5\% level is important because individuals with CFTR mRNA levels of only 5-10\% have normal lung function $(22,23,27)$. However, this high level of Ad vector-derived CFTR expression is transient, lasting somewhere from 4 to 30 days, i.e., this impressive level of expression is linked to the major challenge that expression is limited in time. Although this can be initially overcome by repetitive administration, host defenses eventually limit this strategy, and further repetitive administration does not lead to repetitive expression. Thus, there are advantages and challenges of using Ad vectors to transfer genes in vivo to humans. Ad vectors can deliver amounts of a CFTR cDNA expression cassette that provides amounts of normal CFTR mRNA levels believed to be sufficient to compensate for the airway epithelial deficiency in CFTR function associated with $\mathrm{CF}$, but the present vectors are incapable of providing persistent genetic compensation, and repetitive administration of the same vector is unlikely to compensate for the transient persistence of the vector.

Distribution of vector-derived normal CFTR $m R N A$ in airway epithelium. For the respiratory manifestations of CF to be corrected by gene therapy, the Ad vector must be distributed diffusely across the epithelium, i.e., it is very unlikely that the disease can be corrected by limited "hot spots" of genetic correction (26). In the present study, the "normal" levels of vector-derived CFTR mRNA that were transiently achieved in the airway epithelium in CF using the $\mathrm{Ad}_{\mathrm{GV}} \mathrm{CFTR}$.10 vector represents an average of vectorderived CFTR mRNA levels in approximately 5,000 airway epithelial cells that were analyzed. To evaluate whether the vector was evenly distributed, we attempted to assess the recovered epithelium for the distribution of the $\mathrm{Ad}_{\mathrm{GV}} \mathrm{CFTR} .10$ vector genome using FISH analysis. Although all of the relevant controls were positive, we did not identify any genome "hot spots." However, because of problems with autofluorescence, we could not positively demonstrate that the vector was evenly dispersed. That is, although it is unlikely, we cannot absolutely rule out the possibility that the vector was delivered to a small number of cells expressing at a high level, thus providing a high "average" vector-derived mRNA. There are 2 lines of evidence that make this improbable. First, the device used to deliver the vector provides an even spray, diffusing over the airway epithelium in a limited area (28). Second, the relevant positive controls with cell lines in vitro were easily detectable with this methodology, and the endogenous chromosomes and epithelial cell markers were detected in the brushed airway samples. Thus, it is reasonable to conclude in the context of the expression data that the vector was dispersed in vivo but that the levels per cell were too low to be detected by FISH technology in the context of the background autofluorescence in the clinical samples. 
Lack of effectiveness of repetitive administration. Although repetitive administration of the $\mathrm{Ad}_{\mathrm{GV}} \mathrm{CFTR} .10$ vector to the airway epithelium of individuals with CF appears to be tolerated, this strategy as used in the present study cannot maintain normal levels of vector-derived normal CFTR mRNA in the airway epithelium. In this regard, although vector-derived CFTR cDNA expression in the airway epithelium was achieved with a second administration, this was observed only at intermediate doses, with no expression observed after the third administration. Interestingly, although there are extensive data in experimental animals demonstrating that antivector host immune defenses are responsible for this (36-39, 42-44), repetitive endobronchial administration of an Ad vector was not associated with increases in levels of detectable systemic anti-Ad neutralizing antibodies (10). We do not know whether repetitive administration of the vector to the individuals in the present study increased local (mucosal) anti-Ad antibodies, as the intense neutrophil-dominated inflammation on the airway epithelial surface precluded assessment of this parameter. However, we have assessed normal individuals receiving a similar Ad vector in a similar fashion for mucosal anti-Ad neutralizing antibodies and have found no, or at most low levels of, local neutralizing antibodies (41). Despite this, in the present study we did observe that high levels of vector-derived CFTR mRNA could be achieved in the airway epithelium only in the context of low systemic levels of anti-Ad neutralizing antibodies. Together with the observation that it was not possible to achieve vector-derived expression in the airway epithelium by the third administration, it appears that humans have host defenses that limit persistence as well as defending against effective repetitive administration. However, unlike experimental animals, repetitive administration of the Ad vector to the airways of individuals with CF did not elicit systemic neutralizing antibodies (10). Given that the human lung anti-Ad host defenses are not defined, for now the mechanisms underlying the loss of vector expression in the airway must be considered unknown.

Future directions. The present study demonstrates that it is feasible for gene therapy to safely achieve levels of vector-derived CFTR mRNA in the airway epithelium thought to be sufficient to protect against development of the respiratory manifestations of the disease. However, this high level of expression is transient and cannot be maintained using repetitive administration of the same vector. Together, the present study and studies of the human airway epithelium demonstrating a deficiency of Ad receptors on the apical epithelial surface $(45,46)$ suggest that there are 2 major challenges in extending the observations in the present study: efficiency of entry and persistence. Studies of human airway epithelium in vitro and in experimental animals suggest that the efficiency-of-entry hurdle might be overcome by using alternative means of entering cells, such as targeting to alternative receptors $(47,48)$, or nonspecific enhancement of uptake (49-52). If the effi- ciency of entry of the Ad vector can be enhanced, less vector would be required, and this, in theory, would result in less stimulation of antivector host defenses (38). In regard to the persistence challenge, by modifying the vector genome - for example, by deleting the E2, E4, polymerase, preterminal protein, or single-stranded DNA binding protein genes - or all of the Ad genome (other than the 2 ITRs), or by keeping the E3 genes, it may be possible to reduce host defenses against Ad viral genes expressed at low levels in the target cells, thus enhancing persistence (42, 53-55). Alternatively, enhanced persistence might be achieved by using transient systemic immunosuppression $(38,56)$. Should future studies demonstrate a clear role for mucosal anti-Ad neutralizing antibodies in limiting effective expression after repetitive administration by using alternative serotypes, it may be possible to circumvent anti-Ad neutralizing antibodies $(36,37)$. Finally, despite the encouraging results in the present study regarding the levels of gene expression that can be achieved in the airway epithelium with Ad vectors, it is conceivable that it will not be possible to circumvent the challenges of efficiency of entry and persistence to treat the respiratory manifestations of CF. If so, there is a number of gene delivery vector systems, including liposome, adeno-associated virus, and lentivirus, that are being evaluated for this gene therapy application (17-21, 57-59).

\section{Acknowledgments}

We thank G. N. McElvaney and the clinical fellows of the Division of Pulmonary and Critical Care Medicine of Weill Medical College of Cornell University-New York Presbyterian Hospital for help in carrying out these studies; David Cipolla (Genentech Inc.) for help in designing the spray administration strategy; and Duncan McVay and Douglas D. Brough (GenVec Inc.) for help in constructing the $\mathrm{Ad}_{\mathrm{GV}} \mathrm{CFTR} .10$ vector. These studies were supported, in part, by the National Heart, Lung, and Blood Institute (grant P01 HL5174603); the Cystic Fibrosis Foundation, Bethesda, Maryland, USA; the Will Rogers Memorial Fund, Los Angeles, California, USA; Rockefeller University Hospital (NIH GCRC M01-RR00102); Weill Medical College of Cornell University (NIH GCRC M01-RR0047); Genentech Inc., South San Francisco, California, USA; and GenVec Inc., Rockville, Maryland, USA.

\footnotetext{
1. Kerem, B., et al. 1989. Identification of the cystic fibrosis gene: genetic analysis. Science. 245:1073-1080.

2. Riordan, J.R., et al. 1989. Identification of the cystic fibrosis gene: cloning and characterization of complementary DNA. Science. 245:1066-1073.

3. Rommens, J.M., et al. 1989. Identification of the cystic fibrosis gene: chromosome walking and jumping. Science. 245:1059-1065.

4. Welsh, M.J., Tsui, L.-C., Boat, T.F., and Beaudet, A.L. 1995. Cystic fibrosis. In The metabolic and molecular basis of inherited disease. C.R. Scriver, A.L. Beaudet, W.S. Sly, and D. Valle, editors. McGraw-Hill. New York, NY. 3799-3876

5. Cystic Fibrosis Foundation. 1997. Opening doors to opportunities. New challenges and new interventions. 1997 Annual Report Cystic Fibrosis Foundation. Bethesda, MD. 1-13.

6. Quinton, P.M. 1986. Missing $\mathrm{Cl}$ conductance in cystic fibrosis. Am.J. Physiol. 251:C64-C652.
} 
7. Rosenfeld, M.A., et al. 1992. In vivo transfer of the human cystic fibrosis transmembrane conductance regulator gene to the airway epithelium. Cell. 68:143-155.

8. Crystal, R.G., et al. 1994. Administration of an adenovirus containing the human CFTR CDNA to the respiratory tract of individuals with cystic fibrosis. Nat. Genet. 8:42-51.

9. Bellon, G., et al. 1997. Aerosol administration of a recombinant adenovirus expressing CFTR to cystic fibrosis patients: a phase I clinical trial. Hum. Gene Ther. 8:15-25.

10. Harvey, B.-G., et al. 1999. Variability of human systemic humoral immune responses to adenovirus gene transfer vectors administered to different organs. J. Virol. 73:6729-6742.

11. Hay, J.G., McElvaney, N.G., Herena, J., and Crystal, R.G. 1995. Modification of nasal epithelial potential differences of individuals with cystic fibrosis consequent to local administration of a normal CFTR cDNA adenovirus gene transfer vector. Hum. Gene Ther. 6:1487-1496.

12. Knowles, M.R., et al. 1995. A controlled study of adenoviral-vector-mediated gene transfer in the nasal epithelium of patients with cystic fibrosis. N. Engl. J. Med. 333:823-831.

13. Zabner, J., et al. 1993. Adenovirus-mediated gene transfer transiently corrects the chloride transport defect in nasal epithelia of patients with cystic fibrosis. Cell. 75:207-216.

14. Zabner, J., et al. 1996. Repeat administration of an adenovirus vector encoding cystic fibrosis transmembrane conductance regulator to the nasal epithelium of patients with cystic fibrosis. J. Clin. Invest. 97:1504-1511.

15. Zuckerman, J.B., et al. 1999. A phase I study of adenovirus-mediated transfer of the human cystic fibrosis transmembrane conductance regulator gene to a lung segment of individuals with cystic fibrosis. Ameri can Society of Gene Therapy, Program of the 2 nd Annual Meeting. Washington, DC. June 9-13, 1999. p. 121a.

16. Perricone, M.A., et al. 1997. Molecular analysis of adenovirus-mediated transfer and expression efficiency of CFTR CDNA in individuals with CF. Pediatr. Pulmonol. 14:261. (Abstr.)

17. Caplen, N.J., et al. 1995. Liposome-mediated CFTR gene transfer to the nasal epithelium of patients with cystic fibrosis. Nat. Med. 1:39-46.

18. Gill, D.R., et al. 1997. A placebo-controlled study of liposome-mediated gene transfer to the nasal epithelium of patients with cystic fibrosis. Gene Ther. 4:199-209.

19. Southern, K.W., et al. 1997. Repeated nasal administration of liposomemediated CFTR gene transfer reagents: the clinical and immunological consequences. Pediatr. Pulmonol. 14:A209. (Abstr.)

20. Stern, M., et al. 1999. A double blind placebo controlled trial of pulmonary and nasal administration of liposome-mediated CFTR gene transfer in CF subjects. Am. J. Respir. Crit. Care Med. 157:A564. (Abstr.)

21. Wagner, J.A., et al. 1998. Efficient and persistent gene transfer of AAVCFTR in maxillary sinus. Lancet. 351:1702-1703.

22. Chu, C.S., et al. 1991. Variable deletion of exon 9 coding sequences in cystic fibrosis transmembrane conductance regulator gene mRNA transcripts in normal bronchial epithelium. EMBOJ. 10:1355-1363.

23. Chu, C.S., Trapnell, B.C., Curristin, S.M., Cutting, G.R., and Crystal, R.G. 1992. Extensive posttranscriptional deletion of the coding sequences for part of nucleotide-binding fold 1 in respiratory epithelial mRNA transcripts of the cystic fibrosis transmembrane conductance regulator gene is not associated with the clinical manifestations of cystic fibrosis. J. Clin. Invest. 90:785-790.

24. Dorin, J.R., et al. 1996. A demonstration using mouse models that successful gene therapy for cystic fibrosis requires only partial gene correction. Gene Ther. 3:797-801.

25. Goldman, M.J., Yang, Y., and Wilson, J.M. 1995. Gene therapy in a xenograft model of cystic fibrosis lung corrects chloride transport more effectively than the sodium defect. Nat. Genet. 9:126-131.

26. Johnson, L.G., et al. 1992. Efficiency of gene transfer for restoration of normal airway epithelial function in cystic fibrosis. Nat. Genet. 2:21-25.

27. Cutting, G.R., et al. 1990. Two patients with cystic fibrosis, nonsense mutations in each cystic fibrosis gene, and mild pulmonary disease. $N$. Engl. J. Med. 323:1685-1689.

28. Sweeney, T.D., Cipolla, D., and Gonda, I. 1995. Localized delivery of particles to a small region of the airways. Am. J. Respir. Crit. Care Med. 151:A61. (Abstr.)

29. Danel, C., Erzurum, S.C., McElvaney, N.G., and Crystal, R.G. 1996. Quantitative assessment of the epithelial and inflammatory cell populations in large airways of normals and individuals with cystic fibrosis. Am. J. Respir. Crit. Care Med. 153:362-368.

30. Wang, A.M., Doyle, M.V., and Mark, D.F. 1989. Quantitation of mRNA by the polymerase chain reaction. Proc. Natl. Acad. Sci. USA. 86:9717-9721.

31. Trapnell, B.C., et al. 1991. Expression of the cystic fibrosis transmembrane conductance regulator gene in the respiratory tract of normal individuals and individuals with cystic fibrosis. Proc. Natl. Acad. Sci. USA. 88:6565-6569.

32. Yoshimura, K., et al. 1991. Expression of the cystic fibrosis transmembrane conductance regulator gene in cells of non-epithelial origin. Nucleic Acids Res. 19:5417-5423.
33. Gussoni, E., et al. 1996. A method to codetect introduced genes and their products in gene therapy protocols. Nat. Biotechnol. 14:1012-1016.

34. Hersh, J., Crystal, R.G., and Bewig, B. 1995. Modulation of gene expression after replication-deficient, recombinant adenovirus-mediated gene transfer by the product of a second adenovirus vector. Gene Ther. 2:124-131.

35. Brezillon, S., et al. 1997. Decreased expression of the cystic fibrosis transmembrane conductance regulator protein in remodeled airway epithelium from lung transplanted patients. Hum. Pathol. 28:944-952.

36. Mack, C.A., et al. 1997. Circumvention of anti-adenovirus neutralizing immunity by administration of an adenoviral vector of an alternate serotype. Hum. Gene Ther. 8:99-109.

37. Mastrangeli, A., et al. 1996. "Sero-switch" adenovirus-mediated in vivo gene transfer: circumvention of anti-adenovirus humoral immune defenses against repeat adenovirus vector administration by changing the adenovirus serotype. Hum. Gene Ther. 7:79-87.

38. Wilson, J.M. 1996. Adenoviruses as gene-delivery vehicles. N. Engl. J. Med. 334:1185-1187.

39. Yang, Y., Li, Q., Ertl, H.C., and Wilson, J.M. 1995. Cellular and humoral immune responses to viral antigens create barriers to lung-directed gene therapy with recombinant adenoviruses. J. Virol. 69:2004-2015.

40. McElvaney, N.G., and Crystal, R.G. 1995. IL-6 release and airway administration of human CFTR cDNA adenovirus vector. Nat. Med. 1:182-184.

41. Harvey, B.-G., Hackett, N.R., and Crystal, R.G. 1999. Airway administration of first generation adenovirus vectors to normal individuals. Am.J. Respir. Crit. Care Med. 15:A433. (Abstr.)

42. Harrod, K.S., Hermiston, T.W., Trapnell, B.C., Wold, W.S., and Whitsett, J.A. 1998. Lung-specific expression of adenovirus E3-14.7K in transgenic mice attenuates adenoviral vector-mediated lung inflammation and enhances transgene expression. Hum. Gene Ther. 9:1885-1898.

43. Kaplan, J.M., et al. 1997. Characterization of factors involved in modulating persistence of transgene expression from recombinant adenovirus in the mouse lung. Hum. Gene Ther. 8:45-56.

44. Peng, Y., et al. 1999. Inhibition of tumor necrosis factor alpha by an adenovirus-encoded soluble fusion protein extends transgene expression in the liver and lung [In Process Citation]. J. Virol. 73:5098-5109.

45. Pickles, R.J., et al. 1998. Limited entry of adenovirus vectors into well-differentiated airway epithelium is responsible for inefficient gene transfer. J. Virol. 72:6014-6023.

46. Walters, R.W., et al. 1999. Basolateral localization of fiber receptors limits adenovirus infection from the apical surface of airway epithelia. J. Biol. Chem. 274:10219-10226.

47. Dmitriev, I., et al. 1998. An adenovirus vector with genetically modified fibers demonstrates expanded tropism via utilization of a coxsackievirus and adenovirus receptor-independent cell entry mechanism. J. Virol. 72:9706-9713.

48. Wickham, T.J., Roelvink, P.W., Brough, D.E., and Kovesdi, I. 1996. Adenovirus targeted to heparan-containing receptors increases its gene delivery efficiency to multiple cell types. Nat. Biotechnol. 14:1570-1573.

49. Katkin, J.P., Husser, R.C., Langston, C., and Welty, S.E. 1997. Exogenous surfactant enhances the delivery of recombinant adenoviral vectors to the lung. Hum. Gene Ther. 8:171-176.

50. Lee, J.H., Zabner, J., and Welsh, M.J. 1999. Delivery of an adenovirus vector in a calcium phosphate coprecipitate enhances the therapeutic index of gene transfer to airway epithelia. Hum. Gene Ther. 10:603-613.

51. Worgall, S., Singh, R., Worgall, T., and Crystal, R.G. 1999. Free cholesterol enhances adenoviral vector gene transfer to CAR-deficient cells in vitro. American Society of Gene Therapy, Program of the 2 nd Annual Meeting. Washington, DC. June 9-13. 199:131a.

52. Chu, Q., et al. 1999. Enhancement of adenovirus-mediated gene transfer to columnar epithelial cells in mouse trachea by EGTA. American Society of Gene Therapy, Program of the 2nd Annual Meeting. Washington, DC. June 9-13. 199:116a. (Abstr.)

53. Amalfitano, A., and Chamberlain, J.S. 1997. Isolation and characterization of packaging cell lines that coexpress the adenovirus E1, DNA polymerase, and preterminal proteins: implications for gene therapy. Gene Ther. 4:258-263.

54. Armentano, D., et al. 1997. Effect of the E4 region on the persistence of transgene expression from adenovirus vectors. J. Virol. 71:2408-2416.

55. Engelhardt, J.F., Litzky, L., and Wilson, J.M. 1994. Prolonged transgene expression in cotton rat lung with recombinant adenoviruses defective in E2a. Hum. Gene Ther. 5:1217-1229.

56. Jooss, K., Turka, L.A., and Wilson, J.M. 1998. Blunting of immune responses to adenoviral vectors in mouse liver and lung with CTLA4Ig. Gene Ther. 5:309-319.

57. Porteous, D.J., et al. 1997. Evidence for safety and efficacy of DOTAP cationic liposome mediated CFTR gene transfer to the nasal epithelium of patients with cystic fibrosis. Gene Ther. 4:210-218.

58. Flotte, T.R., and Carter, B.J. 1998. Adeno-associated virus vectors for gene therapy of cystic fibrosis. Methods Enzymol. 292:717-732.

59. Goldman, M.J., Lee, P.S., Yang, J.S., and Wilson, J.M. 1997. Lentiviral vectors for gene therapy of cystic fibrosis. Hum. Gene Ther. 8:226-2268. 